\title{
The unmanned aircraft guide parking solution
}

\author{
Li Yongdong, Rohde \& Schwarz China, Specialist of product \& system department
}

\begin{abstract}
This article gives us the unmanned aircraft guide parking solution. we can only use UWB devices and drones to realize the complex cooperation between cars, park, wireless communication system, let it become easy to set up.

Index Terms- parking, UWB, unmanned aircraft, accurate location, vehicle scheduling, not cellular system, short distance wireless communication, easy to set up, parking solution, Location pilot, Location point, Entrance pilot, Route pilot, Drone, Car point.
\end{abstract}

\section{INTRODUCTION}

A we know, when there are a lot of cars want to park in a parking lot having a large area, it is very difficult to find the accurate location for every car in a short time, so that a lots of cars have to be drove round and round.

It makes the parking lot becomes heavily congested. We can only use UWB devices and drones to realize guiding cars to empty stalls function.

There is not complex cellular system and local area network, so that it is easy to set up and do not need the right using the frequency band.

Not only it can be used in parking lot, but also in storage , vehicle, post mail deliver scheduling.

\section{ABBREVIATIONS AND ACRONYMS}

LI-Location pilot

-It is an UWB device, is installed on the pillars, monitors the stall if empty or occupied.

LO-Location point

-It is an UWB device, is installed on the ground of stalls' center.

CO-Car point

-It is an UWB device and it is put on the car's roof when the car enters the gate of the parking lot. It is with one unique index.

EI-Entrance pilot

-It is an UWB device, is installed beside the door of entryway. it is responsible for defining one unique index to some car and notices the index to the drone which will guide the car to empty stall.

RI-Route pilot
-It is an UWB device, is installed on the ceiling above the roads, it is route pilot type and with unique index. It will always broadcast its index, it is for drones' navigation in door. Drone

- One UWB device and a set of cameras are installed on it

- It can get the empty stall location (x,y) from Lis

- It can save the empty location (x,y)

- It can find the way under the help of RIs

- It can get $\mathrm{CO}$ index need to guide from EI

- It can communicate with the $\mathrm{CO}$ need to be guided

- It can check the distance between CO by UWB way

- It can avoid crashing by camera anf UWB ways

- It can fly to charging station by itself

\section{THE PROCESS OF PARKING}

\section{A. LP set up the $X-Y$ coordinate system}

One LI covers one area, covers a few of stalls. for example, see figure 1:

- This LP cover 4 stalls $(\mathrm{x}, \mathrm{y}),(\mathrm{x}+1, \mathrm{y}),(\mathrm{x}, \mathrm{y}+1),(\mathrm{x}+1, \mathrm{y}+1)$

- Red points are Los, they are always broadcast their locations $(\mathrm{x}, \mathrm{y}),(\mathrm{x}+1, \mathrm{y}),(\mathrm{x}, \mathrm{y}+1),(\mathrm{x}+1, \mathrm{y}+1)$.

- When one car parks one location $(\mathrm{x}, \mathrm{y})$, the connection between LI and LO on the location (x,y) is broken. LI sets $\operatorname{status}[\mathrm{x}, \mathrm{y}]=1$, otherwise it is empty, sets status $[\mathrm{x}, \mathrm{y}]=0$.

- So all of stalls' locations can be defined by (x,y), and stalls' status can be known from status[x,y] of LI.

- LI broadcasts the empty location (x,y), Drones near the LI can get this message and the drone will save $(\mathrm{x}, \mathrm{y})$ for next car guide.

- After the drone saves the (x,y), it will notice LI to lock the location $(\mathrm{x}, \mathrm{y})$ and not to broadcast $(\mathrm{x}, \mathrm{y})$ is empty any more. 


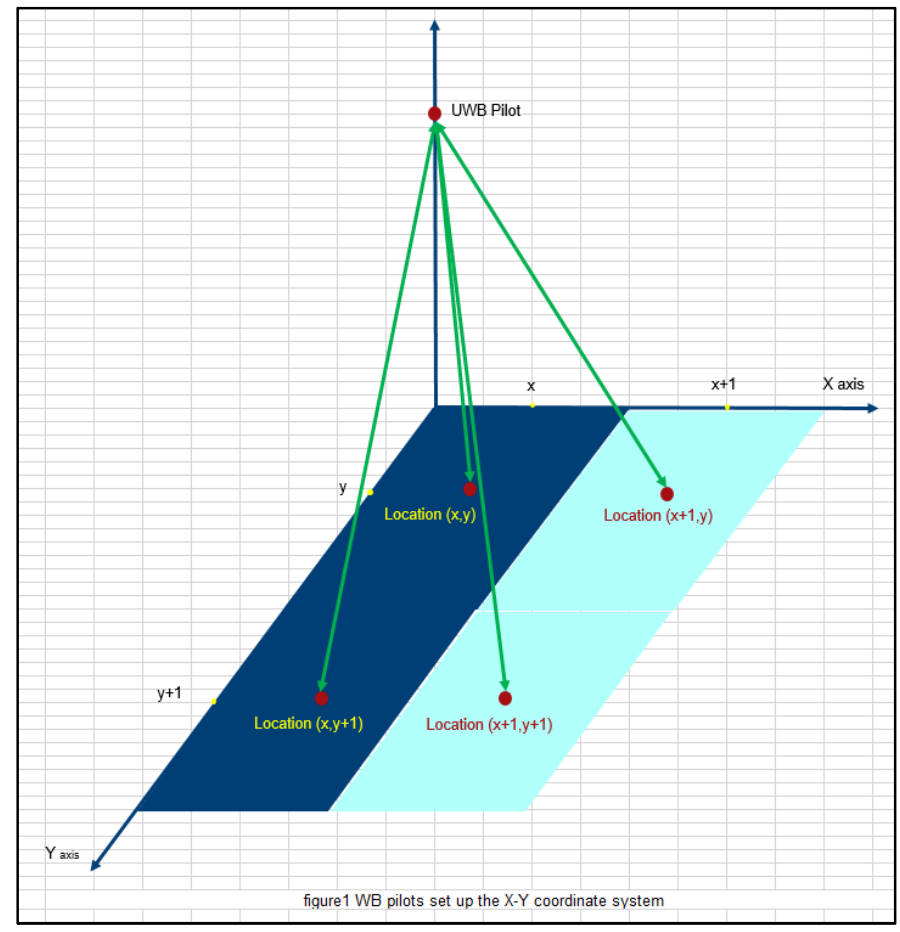

B. The drone finds the accurate empty location

The drone flies according to the route defined in advance. There are RIs broadcasting special index and route type on the routes. The drone can get the direction by capturing the RIs' broadcasting indexes.

At the same time, it also captures the empty location $(\mathrm{x}, \mathrm{y})$ from LI close to it. it will double check if the stall is empty really by camera. If yes, $\mathrm{C}$ step.

\section{Lock the location}

The drone sends the confirmation to LI. LI lock $(\mathrm{x}, \mathrm{y})$ in its record. The LI does not broadcast $(\mathrm{x}, \mathrm{y})$ any more. The drone saves the location $(\mathrm{x}, \mathrm{y})$.

\section{The drone flies back to the entrance}

The drone flies back to entrance. The first car has gotten the $\mathrm{CO}$ with the unique index. The drone also gets the same index from $\mathrm{EI}$.

\section{E. The drone binds some car at the entrance}

The drone binds the $\mathrm{CO}$ with same index by communicating to each other.

\section{F. Guide the car}

The drone flies in front of the car. It flies according to the way close to $(\mathrm{x}, \mathrm{y})$. It always checks the distance from car, so that it can be followed by the car.

The drone flies above the empty stall $(\mathrm{x}, \mathrm{y})$ location until it cannot capture the broadcasting signal from LO.

During flying, the drones have to avoid crashing by cameras and UWB checking.

\section{G. Parking}

When LI finds the connection with $\mathrm{LO}(\mathrm{x}, \mathrm{y})$ is broken, the LI sets status $[\mathrm{x}, \mathrm{y}]=1$, also unlock the location.

When the drone does not get the broadcasting from the LO $(\mathrm{x}, \mathrm{y})$, it also clear all records for this issue, restarts step B.

\section{H. Unlock the location after the car leaves}

When the car leaves the stall, the LI will capture the broadcasting from $\mathrm{LO}(\mathrm{x}, \mathrm{y})$, the $\mathrm{LI}$ sets status $[\mathrm{x}, \mathrm{y}]=0$, and broadcasts that $(\mathrm{x}, \mathrm{y})$ is empty again.

\section{Example}

We give an example: from the figure 2, we can see the locations of LI, LO, EI, RI, CO ,Drones in X-Y coordinate system.

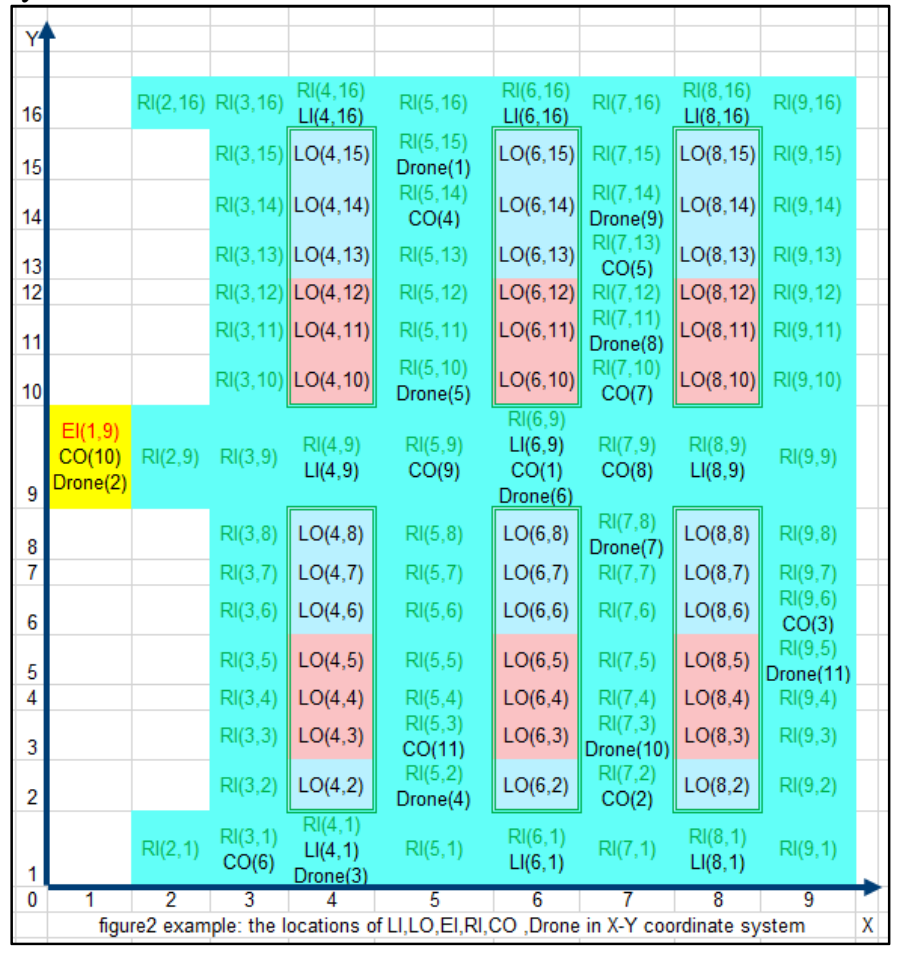

\section{ADVANCE}

\section{A. No server}

As we known, for improving parking efficiency, it is difficult for user to setup a server and a local network to connect all of UWB clients, but it will be much easier than before like next:

1. Install LIs on the pillars and install LOs on the ground for every stall and adjust Lis' UWB antennas so that LIs can setup the connection with the LOs.

2. Set the unique locations $(\mathrm{x}, \mathrm{y})$ to their corresponding LIs and the empty $(\mathrm{x}, \mathrm{y}) \mathrm{s}$ are broadcasted.

3. Install RIs on the ceiling above the route and set the pilots to route type and set different indexes for the RIs, so that drones can follow the indexes to different locations.

4. Install the EI beside the entrance gate, it can broadcast next $\mathrm{CO}$ index need to be guided.

5. When car enters the parking area, it can get one $\mathrm{CO}$ with one unique index from EI and it is put on the top of the car. At the same time, the EI broadcasts the index, the drone preparing to guide will get it from EI, then the drone contacts with the $\mathrm{CO}$ by the index. They binds together and the car will be guide by the drone. 


\section{B. No LAN}

1. Need not to set up local network with cables.

C. Easy to set up

1. After installing the pilots and points, it can work.

D. Low cost

1. Only need UWB devices and drones, it's coast should be low.

\section{E. High isolation}

1. UWB devices should use the higher frequency band, like channel $9,9484.8 \mathrm{MHz}$, then the fading will be faster, the coverage of UWB signal is not wide, so the solution gets high isolation, interference will be lower.

\section{F. NO diffraction}

1. As the frequency of pilots is about $10 \mathrm{GHz}$, its wave length is about $3 \mathrm{~mm}$, there is not diffraction.

2. When the stall is empty, the connection between LIs and LOs can be setup, when it is not empty, the connection is broken. It is very easy to get the stall status.

G. Low interference

1. The time of UWB pulse is only about $2 n s$, if the interval of UWB data transmit is $1 \mathrm{~s}$, the impact probability is only $10^{-6}$, the interference in time domain is low.

\section{H. High capacity}

1. As low interference, the solution can support up to $10^{6}$ pairs of UWB devices in the same coverage, so the solution can support lots of drones ,LIs pilots, RIs, COs, and EIs.

\section{CONCLUSION}

\section{As UWB frequency is about $10 \mathrm{GHz}$, the signal is}

millimeter wave, the fading of signal is faster, so that the interference is lower.

Also the band wide of UWB is over $500 \mathrm{MHz}$, the period of UWB pulse is about $1 \mathrm{~ns}$, the capacity can be higher.

And UWB signal is millimeter wave, we can easy to know the stall status by checking the connection between two UWB devices.

UWB devices support broadcasting and communication to each other by MAC addresses, and also support inputting the UWB device types, index, and location $(\mathrm{x}, \mathrm{y})$ to the payload of UWB message to broadcast, so that

1. UWB devices types can be recognized quickly: EI, CO, Drone, RI, LI or LO.

2. It can be realized that LIs broadcast empty stall location $(\mathrm{x}, \mathrm{y})$, drones can get the information for parking.

3. Drone can find the EI and gets the $\mathrm{CO}$ index need to be guided quickly.
4. Drones can guide cars to the empty stalls under RIs' help.

Then, no server, not LAN, easy to setup, low cost, high isolation, no diffraction, low interference, high capacity will become this solution advantages.

\section{REFERENCES}

【1】 Ecma International, < ECMA-368, High Rate Ultra Wideband PHY and MAC Standard $>$

【2】 IEEE, $<802.15 .4^{\mathrm{TM}}-2020$, IEEE Standard for Low Rate Wireless Networks $>$

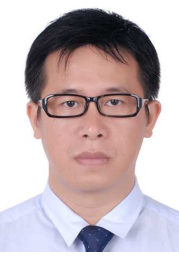

Only Author Li Yongdong, was born in FuChuan,WuZhou, GX, China in 1970. Rohde \& Schwarz China, Specialist of product \& system department, graduated from Sun Yat-sen University and University of Electronic Science and Technology of China. 